\title{
Validação dos marcadores da vulnerabilidade em saúde da pessoa com insuficiência cardíaca
}

\author{
Validation of health vulnerability markers for people with heart failure \\ Validación de marcadores de vulnerabilidad en salud de las personas con insuficiencia \\ cardíaca
}

Virna Ribeiro Feitosa Cestari ${ }^{1 *}$, Raquel Sampaio Florêncio ${ }^{1}$, Vera Lúcia Mendes de Paula Pessoa ${ }^{1}$, Thereza Maria Magalhães Moreira ${ }^{1}$.

\section{RESUMO}

Objetivo: Validar os marcadores de Vulnerabilidade em Saúde (VS) de pessoas com Insuficiência Cardíaca (IC). Métodos: Estudo metodológico, constituído pela identificação dos marcadores da VS da pessoa com IC, construção das suas definições constitutivas e operacionais e validação de conteúdo por especialistas. Participaram da validação 19 juízes, profissionais de saúde, com experiência clínica, pesquisas e publicações sobre a temática, provenientes de cinco estados brasileiros (Ceará, Bahia, São Paulo, Rio de Janeiro e Santa Catarina). Para análise da concordância e confiabilidade calculou-se o Índice de Validade de Conteúdo (IVC) e o Teste Binomial. Resultados: Foram validados 49 marcadores, agrupados em subdimensões e, em seguida, organizados em três dimensões: Pessoa humana (situação socioeconômica e demográfica, perfil clínico, aprendizagem, comportamentos de saúde, saúde mental), Co-presenças (apoio social, vínculos familiares) e Cuidado (tecnologias em saúde, custos de saúde e serviços de saúde). Todos apresentaram IVC excelentes $(\geq 0,78)$ e $p>0,05$. Conclusão: Os marcadores operacionalizaram o fenômeno VS, pois apresentaram parâmetros adequados de validade. Assim, podem subsidiar a construção de instrumentos de pesquisa e cuidado.

Palavras-chave: Insuficiência cardíaca, Vulnerabilidade em saúde, Cuidados de enfermagem, Psicometria, Estudos de validação.

\begin{abstract}
Objective: To validate the Health Vulnerability (HV) markers of people with Heart Failure (HF). Methods: Methodological study, consisting of the identification of the HV markers of the person with HF, construction of their constitutive and operational definitions and content validation by specialists. Nineteen judges, health professionals, with clinical experience, research and publications on the subject, from five Brazilian states (Ceará, Bahia, São Paulo, Rio de Janeiro and Santa Catarina) participated in the validation. For the analysis of agreement and reliability, the Content Validity Index (CVI) and the Binomial Test were calculated. Results: 49 markers were validated, grouped into subdimensions and then organized into three dimensions: Human person (socioeconomic and demographic situation, clinical profile, learning, health behaviors, mental health), Co-presences (social support, family ties) and Care (health technologies, health costs and health services). All had excellent CVI $(\geq 0.78)$ and $p>0.05$. Conclusion: The markers operationalized the HV phenomenon, as they presented adequate parameters of validity. Thus, they can subsidize the construction of research and care instruments.
\end{abstract}

Key words: Heart failure, Health vulnerability, Nursing care, Psychometrics, Validation study.

1 Universidade Estadual do Ceará (UECE), Fortaleza - CE. `E-mail: virna.ribeiro@hotmail.com 


\section{RESUMEN}

Objetivo: Validar los marcadores de Vulnerabilidad en Salud (VS) de personas con Insuficiencia Cardíaca (IC). Métodos: Estudio metodológico, consistente en la identificación de los marcadores de VS de la persona con IC, construcción de sus definiciones constitutivas y operativas y validación de contenido por especialistas. En la validación participaron 19 jueces, profesionales de la salud, con experiencia clínica, investigación y publicaciones sobre el tema, de cinco estados brasileños (Ceará, Bahía, São Paulo, Rio de Janeiro y Santa Catarina). Para el análisis de concordancia y confiabilidad se calculó el Índice de Validez de Contenido (IVC) y la Prueba Binomial. Resultados: Se validaron 49 marcadores, agrupados en subdimensiones y luego organizados en tres dimensiones: Persona humana (situación socioeconómica y demográfica, perfil clínico, aprendizaje, conductas de salud, salud mental), Copresencias (apoyo social, vínculos familiares) y Cuidado (tecnologías sanitarias, costes sanitarios y servicios sanitarios). Todos tenían un IVC excelente $(\geq 0,78)$ yp> 0,05. Conclusión: Los marcadores operacionalizaron el fenómeno VS, ya que presentaron parámetros de validez adecuados. Así, pueden subsidiar la construcción de instrumentos de investigación y atención.

Palabras clave: Insuficiencia cardíaca, Vulnerabilidad en salud, Atención de enfermería, Psicometría, Estudio de validación.

\section{INTRODUÇÃO}

A Vulnerabilidade em Saúde (VS) é concebida como parte da condição humana e, por tal, exprime relação direta com o cuidar por cingir a pluridimensionalidade do ser humano. Em uma acepção ampliada da vulnerabilidade, percebe-se como uma compreensão e transformação de determinado fenômeno de saúde a partir de uma perspectiva individual e sociopolítica (ENANG I, et al., 2019; PANDOVEZE MC, et al., 2019). As definições e aplicações desse conceito na saúde são diversos, pois abrangem múltiplas temáticas, em diferentes níveis de complexidade, com vistas à promoção da saúde (CLARK B e PRETO N, 2018).

$\mathrm{Na}$ enfermagem, a VS tem sido estudada nas mais variadas perspectivas (MAFFACCIOLLI R e OLIVEIRA DLLC, 2018), contudo, constatou-se a carência de pesquisas que abordem a vulnerabilidade em saúde da pessoa com insuficiência cardíaca (IC). A IC é uma cardiopatia grave que se destaca por ser um desafiador problema de saúde pública, pois, apesar dos avanços na terapia cardíaca, tem caráter progressivo, é altamente sintomática e possui elevado risco de mortalidade precoce, que exige dos indivíduos acometidos, familiares/cuidadores e gestores de serviços de saúde comprometimento para prevenção das complicações dessa síndrome (SEFEROVIC PM et al., 2020; FERNANDES ADF, et al., 2020).

O impacto e a interferência negativa da IC na vida das pessoas são notáveis; pessoas com IC sofrem modificações em seu padrão de vida normal, pois a doença acarreta prejuízos tanto no desempenho físico quanto no emocional dos pacientes, contribuindo para a redução/piora da expectativa e qualidade de vida (LI X, 2019; YU DSF et al., 2019). Urge, portanto, o planejamento de ações de saúde que abarque aspectos relevantes da vulnerabilidade dessa população. Neste estudo, o conceito de vulnerabilidade adotado partiu da proposta que a compreende como fenômeno multidimensional, contextual e dinâmico, intrínseco à existência humana, resultante da interação entre Pessoa humana, Co-presenças e Cuidado, que acarreta agravos à saúde ou possibilita a evolução do indivíduo ou coletividade (CESTARI VRF, et al., 2017).

Inúmeros aspectos exacerbam a vulnerabilidade da pessoa com IC. Neste ínterim, conhecer os elementos essenciais ou marcadores que capturem as facetas da vulnerabilidade da pessoa com IC enseja cuidados no intuito de promover saúde. O termo marcador tem seu sentido ligado a uma palavra que o qualifica. São elementos cuja presença ou ausência definem e caracterizam a dimensão da vulnerabilidade, compreendendo a interação de atributos subjetivos e contextuais na produção do processo saúde-doença como fenômenos sociais e históricos (SILVA DI, et al., 2018).

É valido destacar a inexistência de artigos científicos nacionais e internacionais que abordem a identificação de marcadores de pessoas com IC em situação de vulnerabilidade, evidenciando uma lacuna de conhecimento. Espera-se que esta pesquisa possibilite erigir e operacionalizar o conceito da 
vulnerabilidade nessa população, pois se assume que a vulnerabilidade esteja ancorada em dimensões singulares. Ademais, elencar marcadores nessa área poderá ancorar o cuidado clínico da enfermagem cardiovascular na promoção da saúde e prevenção de agravos, favorecendo a qualidade assistencial. Ante ao exposto, objetivou-se neste estudo validar os marcadores de vulnerabilidade em saúde de pessoas com Insuficiência Cardíaca (IC).

\section{MÉTODOS}

Estudo metodológico, fundamentado na Psicometria, a qual descreve três polos para a construção de instrumentos: teórico, empírico e analítico (PASQUALI L, 2013). A construção deste artigo descreve o polo teórico, constituído de três fases: 1) identificação dos marcadores vulnerabilidade em saúde da pessoa com IC; 2) construção das definições constitutivas e operacionais dos marcadores e 3) validação de conteúdo dos marcadores por especialistas.

Na primeira fase da construção, realizou-se uma revisão integrativa $(\mathrm{RI})$ da literatura, no intuito de elucidar a magnitude do construto vulnerabilidade da pessoa com IC. Para tal, a revisão foi construída a partir do estabelecimento da hipótese ou questão da pesquisa; amostragem ou busca na literatura; seleção e categorização dos estudos; síntese do conhecimento ou apresentação da revisão; interpretação dos resultados; e avaliação dos estudos incluídos na revisão (MENDES KDS, et al., 2019).

No estabelecimento da questão norteadora a estruturação da questão fundamentou-se na estratégia PICO, onde P (população): pessoas com IC; I (interesse): marcadores de vulnerabilidade; C (comparação): não tem; $O$ (resultados): vulnerabilidade em saúde. Assim, a revisão procurou responder à seguinte pergunta: que marcadores compõem a vulnerabilidade em saúde da pessoa com IC?

Realizou-se uma busca pareada no mês de janeiro de 2017, nas bases de dados da saúde Literatura Latino-Americana e do Caribe em Ciências da Saúde (LILACS) e Índice Bibliográfico Espanhol de Ciências da Saúde (IBECS), via Biblioteca Virtual da Saúde e Scientific Electronic Library Online (SciELO); e Cummulative Index to Nursing and Allied Health Literature (CINAHL), SciVerse Scopus e no portal PubMed, via National Library of Medicine.

Para a busca dos artigos foram utilizados os descritores heart failure e health vulnerability, de acordo com a terminologia Medical Subject Headings (MeSH), da PubMed, e a palavra-chave vulnerability, no intuito de ampliar os achados. Para sistematizar as buscas, foi elaborada a seguinte equação: (((heart failure[MeSH Terms]) AND (health vulnerability[MeSH Terms]) OR (vulnerability).

Os critérios de inclusão foram: estudos que possibilitassem a identificação dos marcadores de vulnerabilidade da pessoa com IC; publicados no período de janeiro de 2012 a janeiro de 2017; de livre acesso e disponíveis na íntegra; em língua portuguesa, inglesa ou espanhola. Foram critérios de exclusão: teses, dissertações, comunicação prévia, artigos de revisão ou de reflexão, editoriais, cartas ao editor e trabalhos publicados em anais de eventos. O recorte temporal estabelecido para a seleção dos estudos foi escolhido com base no lançamento da Diretriz Brasileira de Insuficiência Cardíaca Crônica (BOCCHI EA et al., 2012), em janeiro de 2012, tendo em vista que ela reúne as principais evidências anteriores ao ano de sua publicação. Procurou-se, portanto, explorar todo o conhecimento disponível acerca da temática posterior a este período.

Inicialmente, dois pesquisadores independentes realizaram as buscas que identificaram os potenciais estudos primários. Para mostrar o caminho percorrido para a seleção das publicações, utilizou-se o fluxograma Preferred Reporting Items for Systematic reviews and Meta-Analyses (PRISMA) (Figura 1). Assim, a partir dos critérios pré-estabelecidos, foram selecionados e analisados 231 estudos para compor o corpus da revisão. 
Figura 1 - Processo de seleção dos estudos, segundo PRISMA.

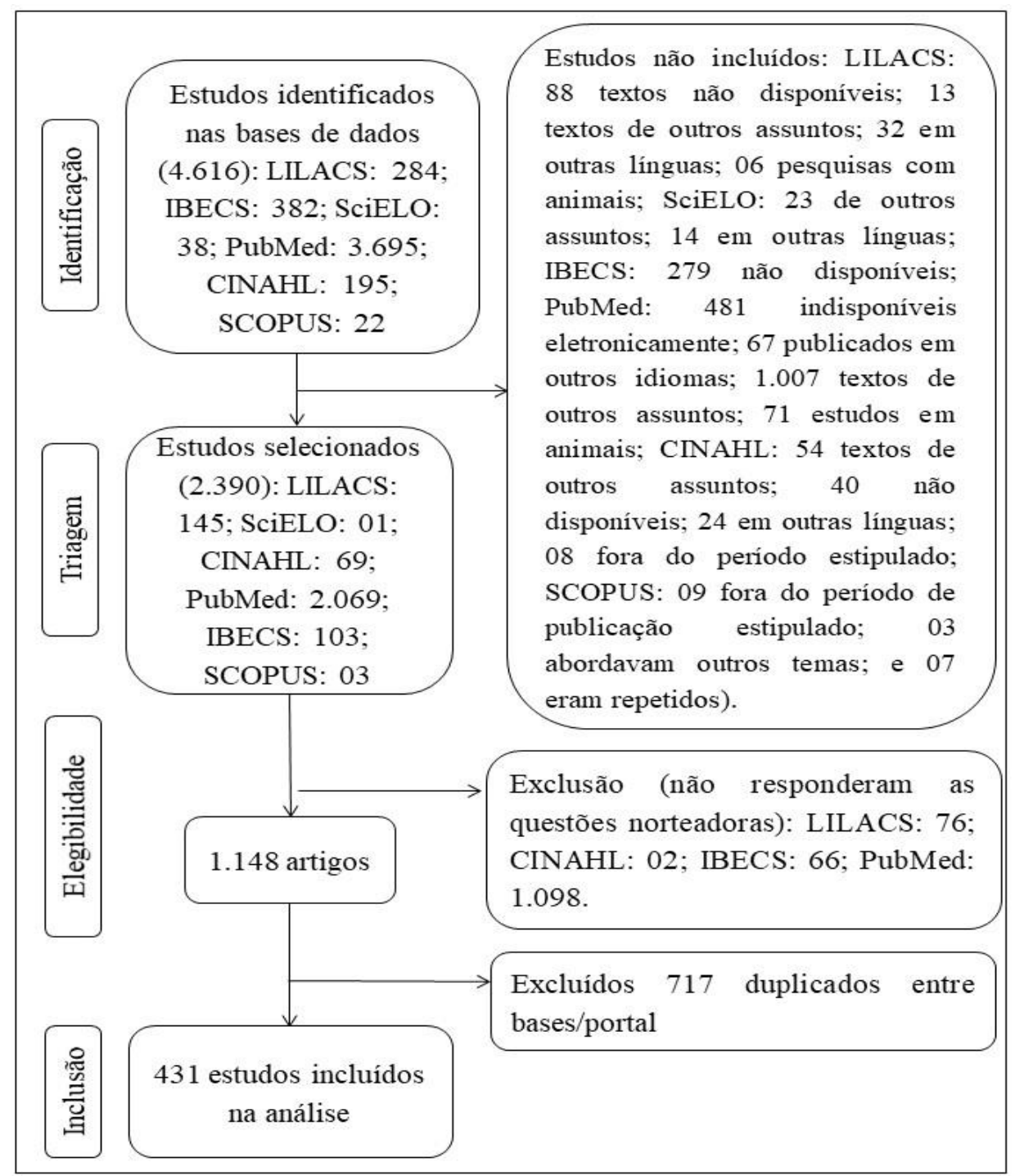

Fonte: Cestari VRF, et al., 2021.

No processo de triagem, os estudos elegíveis foram lidos na íntegra pelos dois revisores, os quais extraíram os dados previamente definidos. Cada pesquisador chegou a uma lista de estudos primários. As duas listas foram comparadas e consolidadas em uma única. Quando houve divergência sobre a inserção ou não de algum artigo na lista final, recorreu-se a um terceiro pesquisador ligado à temática.

$\mathrm{Na}$ extração dos dados dos estudos selecionados, foram explorados: a identificação dos artigos (título, autores, idioma, país, local, periódico e ano); os aspectos metodológicos (delineamento de pesquisa, objetivos, amostragem, tratamento de dados e nível de evidência); análise crítica (resultados e conclusões) e evidências de elementos que comportassem a vulnerabilidade em saúde da pessoa com IC. A partir dos resultados dos estudos, foram identificados os marcadores por meio da categorização de dados semelhantes.

Uma vez realizada essa identificação, procedeu-se a segunda fase da construção, onde foram selecionados os marcadores com potencial para operacionalizar o traço latente "vulnerabilidade em saúde da pessoa com IC". Posteriormente, os dados foram categorizados e recategorizados com base em um processo reflexivo e interpretativo, cujo objetivo foi de construção das definições constitutivas e operacionais.

A definição constitutiva relaciona-se com os conceitos, que são realidades abstratas (como por exemplo o que consta nas definições dos dicionários), e as definições operacionais correspondem à definição do construto por operações concretas (comportamentos ou sensações) (PASQUALI L, 2013). Assim, as 
definições foram extraídas dos artigos analisados, na tentativa de conceber em termos de conceitos próprios da teoria e definir os comportamentos pelos quais o construto se expressa.

$\mathrm{Na}$ terceira fase, foi realizada a validação de conteúdo dos marcadores, definições constitutivas e operacionais por especialistas da área da saúde. A escolha dos especialistas pesquisadores/doutores foi feita por meio de acesso e pesquisa na Plataforma nacional Lattes, da seguinte forma: após acessar o site "Plataforma Lattes", na janela "Currículo Lattes", escolheu-se a opção "Busca", na janela "Buscar Currículo Lattes". O primeiro passo foi escolher o modo de busca, clicando no quadro "Assunto" e, no espaço reservado, escreveu-se "Insuficiência cardíaca", "Questionários" e "Vulnerabilidade em saúde". Em seguida, aplicaramse filtros aos resultados por "Atuação profissional", selecionando-se na Grande área "Ciências da saúde". Não se optou por selecionar área, por compreender que a complexidade da IC demanda conhecimento de diversos profissionais que atuam nos diferentes níveis de atenção à saúde.

Para a seleção dos especialistas, o número de seis a vinte especialistas é o recomendável para o processo de validação (PASQUALI L, 2013). Fizeram parte da validação de conteúdo os especialistas que se adequaram aos critérios: expert o profissional com corpo de conhecimento especializado ou habilidade; extensa experiência no campo específico da prática; níveis altamente desenvolvidos para reconhecimento de padrões e com sua qualidade de expert reconhecida por outros (JASPER MA, 1994).

Aos especialistas foi encaminhada uma carta-convite, via e-mail, explicando objetivo do estudo, método e sua função na pesquisa. Após aceite, foram enviados o Termo de Consentimento Livre e Esclarecido, as instruções para avaliação dos marcadores, com suas definições constitutivas e operacionais, para a validação de conteúdo.

Foram convidados a participar da pesquisa 60 especialistas, dos quais 45 aceitaram, mas apenas 19 responderam ao instrumento de coleta de dados. Assim, a análise de conteúdo foi realizada por 19 especialistas (dez assistenciais e nove docentes/acadêmicos), profissionais de saúde (13 enfermeiros, três educadores físicos, um médico, um nutricionista e um psicólogo), com experiência clínica, pesquisas e publicações sobre a temática ( 17 com expertise em insuficiência cardíaca, um em vulnerabilidade e um em construção de instrumentos em saúde), provenientes de cinco estados brasileiros (Ceará, Bahia, São Paulo, Rio de Janeiro e Santa Catarina).

Os especialistas julgaram a consistência em relação às definições conceituais; a representatividade em relação ao domínio de interesse; a relevância para as interpretações clínicas que poderiam ser feitas com base na sua medida; e a clareza e a possibilidade de compreensão de sua redação (POLIT DF e BECK CT, 2019; FLORÊNCIO RS et al., 2016). Para tal, utilizou-se uma escala categórica ordinal de quatro pontos: 1 o item não é indicativo do construto, 2 - item pouco indicativo do construto, 3 - o item é indicativo do construto e 4 - o item é muito indicativo do construto. Os juízes tiveram um prazo de 15 dias para finalizar tal análise.

Visando a objetividade da validade de conteúdo, calculou-se o Índice de Validade de Conteúdo (IVC), a partir da média do número de respostas "3" e "4" selecionados pelos juízes, por serem consideradas adequadas por representarem o construto em análise. Foi considerado excelente o IVC dos itens (IVCi) maior ou igual a 0,78 e, média de IVC total de 0,90 ou superior (CESTARI VRF, et al., 2019). Foi realizado o teste exato de distribuição binomial, indicado para pequenas amostras, sendo considerados nível de significância p>0,05 e proporção de 0,95 de concordância para estimar a confiabilidade estatística dos IVC.

O presente estudo foi aprovado em Comitê de Ética em Pesquisa (CEP), sob número de parecer 1.962.663. Foram cumpridas todas as normas para pesquisa com seres humanos, presentes na Resolução 466/12 do Conselho Nacional de Saúde do Brasil.

\section{RESULTADOS}

Emergiram 50 marcadores que foram, incialmente, agrupados segundo sua semelhança e, posteriormente, escolheu-se um termo que abrangeu o conjunto dos marcadores, denominados de subdimensões. Em seguida, as subdimensões foram organizadas nas três dimensões: Pessoa humana, Co-presenças e Cuidado (Quadro 1). 
Quadro 1 - Marcadores, subdimensões e dimensões da vulnerabilidade em saúde da pessoa com IC.

\begin{tabular}{|c|c|c|}
\hline Marcadores & Subdimensões & Dimensões \\
\hline Características socioeconômicas e demográficas & $\begin{array}{c}\text { Situação socioeconômica e } \\
\text { demográfica }\end{array}$ & \multirow{30}{*}{$\begin{array}{l}\text { Pessoa } \\
\text { humana }\end{array}$} \\
\hline Classificação funcional & \multirow{9}{*}{ Perfil clínico } & \\
\hline Fração de ejeção & & \\
\hline Sinais e sintomas & & \\
\hline Conjunto de sintomas & & \\
\hline Capacidade funcional & & \\
\hline Fragilidade & & \\
\hline Estado nutricional & & \\
\hline Progressão da doença & & \\
\hline Comorbidades & & \\
\hline Letramento funcional em saúde & \multirow{3}{*}{ Aprendizagem } & \\
\hline Aprendizagem significativa & & \\
\hline Competência cognitiva & & \\
\hline Autocuidado e adesão ao tratamento & \multirow{4}{*}{ Comportamentos de saúde } & \\
\hline Autogestão & & \\
\hline Resolução de problemas & & \\
\hline Estilo de vida & & \\
\hline Autopercepção de saúde & \multirow{13}{*}{ Saúde mental } & \\
\hline Crenças sobre o tratamento & & \\
\hline Espiritualidade & & \\
\hline Bem-estar & & \\
\hline Gratidão & & \\
\hline Otimismo disposicional & & \\
\hline Autoeficácia & & \\
\hline Senso de coerência & & \\
\hline Ativação do paciente & & \\
\hline Aceitação da doença & & \\
\hline Personalidade & & \\
\hline Ansiedade & & \\
\hline Depressão & & \\
\hline Suporte instrumental & \multirow{4}{*}{ Apoio social } & \multirow{7}{*}{$\begin{array}{l}\text { Co- } \\
\text { presenças }\end{array}$} \\
\hline Suporte informacional & & \\
\hline Suporte emocional & & \\
\hline Suporte para a autonomia & & \\
\hline Relações familiares & \multirow{3}{*}{ Vínculos familiares } & \\
\hline Acompanhamento familiar & & \\
\hline Funcionamento familiar & & \\
\hline Tecnologias leves & \multirow{3}{*}{ Tecnologias em saúde } & \multirow{13}{*}{ Cuidado } \\
\hline Tecnologias leve-duras & & \\
\hline Tecnologias duras & & \\
\hline Internações hospitalares & \multirow{5}{*}{ Custos de saúde } & \\
\hline Cuidado formal & & \\
\hline Cuidado informal & & \\
\hline Terapia medicamentosa & & \\
\hline Despesas pessoais & & \\
\hline Acesso aos serviços de saúde & \multirow{5}{*}{ Serviços de saúde } & \\
\hline Confiança no sistema de saúde & & \\
\hline Integralidade do cuidado & & \\
\hline Continuidade relacional & & \\
\hline Alta hospitalar planejada & & \\
\hline
\end{tabular}

Fonte: Cestari VRF, et al., 2021.

Após a elucidação das dimensões, subdimensões e dos marcadores que constituíram o traço latente, foram elaboradas as definições constitutivas e operacionais. Seguiu-se com a validação de conteúdo desses 
conceitos. A dimensão da Pessoa-humana abrangeu cinco subdimensões: situação socioeconômica e demográfica, perfil clínico, aprendizagem, comportamentos de saúde e saúde mental. As definições constitutivas e operacionais foram validadas com IVC de 0,99 e 0,97, respectivamente (Tabela 1).

Tabela 1 - Índice de Validade de Conteúdo e Teste Binomial das definições constitutivas e operacionais dos marcadores da dimensão da Pessoa-humana.

\begin{tabular}{|c|c|c|c|c|}
\hline \multicolumn{5}{|c|}{ Pessoa humana } \\
\hline Marcadores & $\begin{array}{l}\text { IVC Definições } \\
\text { constitutivas }\end{array}$ & $\begin{array}{c}\text { Teste } \\
\text { Binomial }\end{array}$ & $\begin{array}{l}\text { IVC Definições } \\
\text { operacionais }\end{array}$ & $\begin{array}{c}\text { Teste } \\
\text { Binomial }\end{array}$ \\
\hline $\begin{array}{l}\text { Situação socioeconômica e } \\
\text { demográfica }\end{array}$ & 1,0 & - & 1,0 & - \\
\hline $\begin{array}{l}\text { Fatores socioeconômicos e } \\
\text { demográficos }\end{array}$ & 1,0 & 0,377 & 1,0 & 0,377 \\
\hline Perfil clínico & 0,99 & - & 0,97 & - \\
\hline Classificação funcional & 1,0 & 0,377 & 1,0 & 0,377 \\
\hline Fração de ejeção & 1,0 & 0,377 & 1,0 & 0,377 \\
\hline Sinais e sintomas & 1,0 & 0,377 & 1,0 & 0,179 \\
\hline Conjunto de sintomas & 1,0 & 0,377 & 1,0 & 0,377 \\
\hline Capacidade funcional & 1,0 & 0,377 & 1,0 & 0,377 \\
\hline Fragilidade & 0,94 & 0,377 & 0,89 & 0,377 \\
\hline Estado nutricional & 1,0 & 0,377 & 0,84 & 0,377 \\
\hline Progressão da doença & 1,0 & 0,377 & 1,0 & 0,377 \\
\hline Comorbidades & 1,0 & 0,377 & 1,0 & 0,179 \\
\hline Aprendizagem & 1,0 & - & 0,96 & - \\
\hline Letramento funcional em saúde & 1,0 & 0,377 & 1,0 & 0,053 \\
\hline Aprendizagem significativa & 1,0 & 0,377 & 1,0 & 0,377 \\
\hline Competência cognitiva & 1,0 & 0,377 & 0,89 & 0,377 \\
\hline Comportamentos de saúde & 1,0 & - & 0,98 & - \\
\hline $\begin{array}{l}\text { Autocuidado e adesão ao } \\
\text { tratamento }\end{array}$ & 1,0 & 0,377 & 1,0 & 0,377 \\
\hline Autogestão & 1,0 & 0,377 & 1,0 & 0,377 \\
\hline Resolução de problemas & 1,0 & 0,377 & 1,0 & 0,377 \\
\hline Estilo de vida & 1,0 & 0,377 & 0,94 & 0,377 \\
\hline Saúde mental & 0,97 & - & 0,97 & - \\
\hline Autopercepção de saúde & 1,0 & 0,377 & 1,0 & 0,053 \\
\hline Crenças sobre o tratamento & 1,0 & 0,377 & 1,0 & 0,377 \\
\hline Espiritualidade & 1,0 & 0,377 & 1,0 & 0,377 \\
\hline Bem-estar & 1,0 & 0,377 & 1,0 & 0,377 \\
\hline Gratidão & 1,0 & 0,377 & 0,94 & 0,377 \\
\hline Otimismo disposicional & 1,0 & 0,377 & 1,0 & 0,377 \\
\hline Autoeficácia & 0,94 & 0,377 & 1,0 & 0,377 \\
\hline Senso de coerência & 0,78 & 0,377 & 0,78 & 0,377 \\
\hline Ativação do paciente & 0,94 & 0,377 & 1,0 & 0,377 \\
\hline Aceitação da doença & 0,94 & 0,377 & 0,94 & 0,377 \\
\hline Personalidade & 1,0 & 0,377 & 1,0 & 0,377 \\
\hline Ansiedade & 1,0 & 0,011 & 1,0 & 0,011 \\
\hline Depressão & 1,0 & 0,377 & 1,0 & 0,377 \\
\hline IVC total da dimensão & 0,99 & - & 0,97 & - \\
\hline
\end{tabular}

Fonte: Cestari VRF, et al., 2021.

A dimensão das Co-presenças envolveu duas subdimensões: apoio social e apoio familiar. As definições constitutivas e operacionais foram validadas com IVC de 0,98 cada (Tabela 2). 
Tabela 2 - Índice de Validade de Conteúdo e Teste Binomial das definições constitutivas e operacionais dos marcadores da dimensão das Co-presenças.

\begin{tabular}{lcccc}
\hline \multicolumn{1}{c}{ Marcadores } & \multicolumn{2}{c}{ Co-presenças } & & \\
& $\begin{array}{c}\text { Definições } \\
\text { constitutivas }\end{array}$ & $\begin{array}{c}\text { Teste } \\
\text { Binomial }\end{array}$ & $\begin{array}{c}\text { Definições } \\
\text { operacionais }\end{array}$ & Teste Binomial \\
\hline Apoio social & $\mathbf{0 , 9 7}$ & - & $\mathbf{0 , 9 7}$ & - \\
\hline Suporte instrumental & 0,89 & 0,377 & 0,89 & 0,377 \\
Suporte informacional & 1,0 & 0,377 & 1,0 & 0,377 \\
Suporte emocional & 1,0 & 0,377 & 1,0 & 0,377 \\
Suporte para a autonomia & 1,0 & 0,179 & 1,0 & 0,179 \\
\hline Apoio familiar & $\mathbf{1 , 0}$ & - & $\mathbf{1 , 0}$ & - \\
\hline Relações familiares & 1,0 & 0,377 & 1,0 & 0,377 \\
Acompanhamento familiar & 1,0 & 0,377 & 1,0 & 0,377 \\
Funcionamento familiar & 1,0 & 0,377 & 1,0 & 0,377 \\
\hline IVC total da dimensão & $\mathbf{0 , 9 8}$ & - & $\mathbf{0 , 9 8}$ & - \\
\hline
\end{tabular}

Fonte: Cestari VRF, et al., 2021.

A dimensão do Cuidado comportou três subdimensões: tecnologias em saúde, custos com a saúde e serviços de saúde. As definições constitutivas e operacionais foram validadas com IVC de 1,00 e 0,97, respectivamente (Tabela 3).

Tabela 3 - Índice de Validade de Conteúdo e Teste Binomial das definições constitutivas e operacionais dos marcadores da dimensão do Cuidado.

\begin{tabular}{lcccc}
\hline \multicolumn{1}{c}{ Marcadores } & $\begin{array}{c}\text { Cefinições } \\
\text { constitutivas }\end{array}$ & $\begin{array}{c}\text { Teste } \\
\text { Binomial }\end{array}$ & $\begin{array}{c}\text { Definições } \\
\text { operacionais }\end{array}$ & $\begin{array}{c}\text { Teste } \\
\text { Binomial }\end{array}$ \\
\hline Tecnologias em saúde & $\mathbf{1 , 0}$ & - & $\mathbf{0 , 9 8}$ & - \\
\hline Tecnologias leves & 1,0 & 0,377 & 0,94 & 0,377 \\
Tecnologias leve-duras & 1,0 & 0,377 & 1,0 & 0,377 \\
Tecnologias duras & 1,0 & 0,377 & 1,0 & 0,623 \\
\hline Custos com a doença & $\mathbf{1 , 0}$ & - & $\mathbf{0 , 9 7}$ & - \\
\hline Internações hospitalares & 1,0 & 0,377 & 1,0 & 0,377 \\
Cuidado formal & 1,0 & 0,377 & 1,0 & 0,377 \\
Cuidado informal & 1,0 & 0,377 & 1,0 & 0,377 \\
Terapia medicamentosa & 1,0 & 0,377 & 0,89 & 0,377 \\
Despesas pessoais & 1,0 & 0,377 & 1,0 & 0,377 \\
\hline Serviços de saúde & $\mathbf{1 , 0}$ & - & $\mathbf{0 , 9 8}$ & - \\
\hline Acesso aos serviços de saúde & 1,0 & 0,377 & 1,0 & 0,377 \\
Confiança no sistema de saúde & 1,0 & 0,377 & 1,0 & 0,377 \\
Integralidade do cuidado & 1,0 & 0,377 & 1,0 & 0,245 \\
Continuidade relacional & 1,0 & 0,377 & 0,94 & 0,377 \\
Alta hospitalar planejada & 1,0 & 0,377 & 1,0 & 0,377 \\
\hline IVC total da dimensão & $\mathbf{1 , 0}$ & - & $\mathbf{0 , 9 7}$ & - \\
\hline
\end{tabular}

Fonte: Cestari VRF, et al., 2021.

Alguns especialistas solicitaram que o marcador 'Fragilidade' fosse enquadrado dentro do marcador de 'Capacidade funcional', visto que a fragilidade é um estado que envolve a perda de algum domínio do funcionamento humano. Os especialistas também solicitaram a modificação do marcador 'Fatores socioeconômicos e demográficos' para 'Características socioeconômicas e demográficas", pois o termo fator está atrelado ao conceito de risco.

Desta forma, o produto dos marcadores, dimensões e subdimensões da vulnerabilidade em saúde da pessoa com IC apresentou da seguinte forma: na dimensão da Pessoa humana, obtivemos 29 marcadores 
distribuídos em cinco subdimensões; a dimensão das Co-presenças abrangeu sete marcadores em duas subdimensões; e a dimensão do Cuidado apresentou 13 marcadores em três subdimensões.

\section{DISCUSSÃO}

Ao revelar as circunstâncias de vulnerabilidade dessa importante síndrome, avançamos na tentativa de confrontar possíveis determinantes na perspectiva de compreender as dimensões supostamente envolvidas em seu desenvolvimento (FLORÊNCIO RS, et al., 2016). De fato, apreendeu-se que a vulnerabilidade em saúde da pessoa com IC desvela-se a partir dos seus elementos essenciais, de caráter relacional: Pessoa humana, Co-presenças e Cuidado (CESTARI VRF, et al., 2019; SILVA DI et al., 2018). A interação dessas dimensões pode alterar os níveis de vulnerabilidade, interferindo no bem-estar do indivíduo e coletividade.

As dimensões da vulnerabilidade em saúde da pessoa com IC possuem pontos de interseção, o que demonstra a impossibilidade de desvincular a pessoa humana das suas co-presenças e do cuidado, caracterizando uma situação dinâmica e aberta à incorporação de novos elementos, uma vez que a vulnerabilidade assume diversas configurações à medida que os elementos constituintes vão se relacionando (CESTARI VRF, et al., 2017; CESTARI VRF, 2019).

Autores sugerem que a fase vulnerável da IC, característica de pacientes com IC aguda, é definida como o período em que mudanças microambientais no modo de vida, após um episódio descompensação, aumentam o risco para eventos cardiovasculares adversos, como morte e reospitalização por IC. Pacientes que superam essa fase sem intercorrências podem permanecer estáveis por um longo período (YILMAZ MB e MBAZAA A, 2015). Contudo, a estabilidade não exime a necessidade de manutenção de acompanhamento sistemático, assegurando cuidado clínico de enfermagem qualificado.

Embora não haja evidências acerca da relação entre VS e IC, o fato de estudos darem maior foco à situação psicoemocional e física, pode ser explicada por autor da área, pois refere que a vulnerabilidade física dos pacientes é, em muitos casos, acompanhada de vulnerabilidade emocional (BOLDT J, 2019). O diagnóstico de uma condição de doença grave ou a perspectiva de morte pode causar medo, raiva e desespero, sendo o contrário também verdadeiro, pois sujeitos que apresentam vulnerabilidades em sua saúde mental também estão mais suscetíveis a doenças (TIMONET-ANDREU E, et al., 2020).

Essas informações são clinicamente pertinentes. No entanto, no intuito de ampliar os olhares voltados para a IC e aclarar o entendimento da vulnerabilidade nessa população, estabeleceu-se neste estudo uma abordagem que agrupou os marcadores, distribuindo-os em distintas subdimensões que englobam não somente a fase aguda da IC.

A divisão favoreceu a compreensão de que a vulnerabilidade da pessoa com IC não se limita apenas às características individuais, mas abarca suas relações sociais e o cuidado. Desta forma, o cuidado clínico da pessoa com IC deverá considerar as co-presenças, haja vista ser a vulnerabilidade um fenômeno que alcança familiares. Propostas terapêuticas tendem a ser mais exitosas quando pactuadas com todos os atores envolvidos. Isso também foi evidenciado por estudo que clarificou o conceito de VS, onde a dimensão social foi resgatada em torno da situação socioeconômica, programática, violência, acesso aos direitos fundamentais, contexto familiar, identidade demográfica, redes e suportes sociais, cultura, ecossistema, gênero e controle social (FLORÊNCIO RS, 2018).

A construção desses marcadores foi complementada pelo conhecimento de pesquisadores e profissionais da saúde da área com a intenção de validar o conteúdo previamente identificado. Esse tipo de validade verifica a capacidade ou suficiência de um conjunto teórico de informações para medir aquilo que se propõe e, ao envolver um grupo de especialistas, é uma técnica consolidada nos estudos de validação, visto que ajuízam a extensão com que cada item do instrumento representa o fenômeno de interesse (PASQUALI L, 1998; BORGES JWP, et al., 2017).

Essa validação foi realizada por um grupo de especialistas em insuficiência cardíaca/vulnerabilidade em saúde, de diferentes regiões do Brasil, com a finalidade de obter um instrumento com linguagem mais abrangente e superar o viés do regionalismo. Ademais, a seleção de especialistas de diferentes regiões 
permite a abrangência de múltiplas visões, no contexto do país como um todo, considerando a diversidade cultural das dimensões continentais do Brasil, que não podem ser desprezadas (CARVALHO REFL e CASSIANI SHB, 2012).

A validação de conteúdo das definições constitutivas e operacionais dos marcadores elencados obtiveram excelentes IVC e confiabilidade. Esse resultado demonstra que o processo de desvelamento dos marcadores e construção das definições foi fundado em sólido conhecimento, compilado a partir de estudos nacionais e internacionais, o que possibilitou abarcar o traço latente, confirmando-se tal fato na análise dos especialistas.

Estudar uma questão de saúde na perspectiva da vulnerabilidade é buscar um modo de compreender melhor, ou apreender outros modos um problema de saúde (CESTARI VRF, et al., 2017). Ao analisarmos a vulnerabilidade da pessoa com IC a partir das subdimensões e marcadores elencados, percebe-se que há uma ruptura do cuidado tradicional e nos aproximamos da condição humana e das circunstâncias que permeiam a trajetória existencial desses indivíduos. Ademais, a vulnerabilidade pode e deve incluir a vulnerabilidade focada cientificamente; a vulnerabilidade focada na justiça social; a vulnerabilidade focada na ética/empoderamento do paciente; $\mathrm{e}$, a vulnerabilidade focada nas ciências sociais (MERMET-BOUVIER P e WHALEN MD, 2020).

Os caminhos trilhados para apreensão da vulnerabilidade da pessoa com IC demonstram que as ações de saúde devem influenciar práticas que impactam na vida da pessoa humana e sua co-presenças, não de maneira linear, mas em uma perspectiva de distintas direções, onde pessoa humana e co-presenças se articulam. Nesse cenário, para reduzir a vulnerabilidade é preciso haver superação dos obstáculos materiais, culturais e políticos; é preciso empoderar e capacitar para que o indivíduo possa proteger-se das situações que o colocam no processo de vulnerabilidade (CESTARI VRF, et al., 2019).

Abordagens como intersetorialidade e interprofissionalidade ampliam os focos das intervenções do sistema de atenção à saúde, pois articulam setores e profissionais, de forma a contemplar a integralidade dos saber e considerar as vulnerabilidades sociais e coletivas, aumentando a igualdade de acesso para toda população (TIMONET-ANDREU E, et al., 2020).

O desafio da política, gestores e profissionais da saúde é captar os marcadores de vulnerabilidade da pessoa com IC, conseguir compreendê-los para, então, propor projetos terapêuticos singulares para cada situação. Por se relevar como prioridade, requer esforços para formulação e implementação de políticas públicas eficazes em seu controle e para o monitoramento da qualidade assistência volvida à esta clientela (YILMAZ MB e MEBAZAA A, 2019; MERMET-BOUVIER P e WHALEN MD, 2020).

Os marcadores identificados são abrangentes e adequados e sua validação com excelentes IVC e concordância entre os especialistas corroboram com esses achados. Apesar da revisão ampla e da validação dos marcadores, esse estudo se limita à fase teórica, não tendo sido explorado aqui a construção e validação de itens para a construção de um instrumento de medida da VS da pessoa com IC, sugestão para outras pesquisas.

\section{CONCLUSÃO}

A condição de vulnerabilidade se materializa em diferentes dimensões e sua releitura a partir da Pessoa humana, Co-presenças e Cuidado, potencializou a percepção da vulnerabilidade e enlaçou a diversidade e particularidades da pessoa com IC. Os marcadores foram avaliados e apresentaram validade de conteúdo pertinentes para identificação de situações de VS da pessoa com IC, podendo subsidiar a construção de instrumentos de pesquisa e cuidado. A compreensão dos marcadores da vulnerabilidade em saúde da pessoa com IC poderá orientar cuidados individualizados e permitirá a construção de instrumentos de mensuração e identificação de novos diagnósticos de enfermagem.

\section{AGRADECIMENTOS E FINANCIAMENTO}

Agradecimentos ao Programa de Pós-graduação Cuidados Clínicos em Enfermagem e Saúde. 


\section{REFERÊNCIAS}

1. BOCCHI EA, et al. Sociedade Brasileira de Cardiologia. Atualização da Diretriz Brasileira de Insuficiência Cardíaca Crônica. Arq Bras Cardiol. 2012; 98(1 supl 1):1-33.

2. BOLDT J. The concept of vulnerability in medical ethics and philosophy. Philos Ethics Humanit Med. 2019; 14:6.

3. BORGES JWP, et al. Nursing Care Interpersonal Relationship Questionnaire: elaboration and validation. Rev. LatinoAm. Enfermagem. 2017;25:e2962.

4. CARVALHO REFL, CASSIANI SHB. Cross-cultural adaptation of the Safety Attitudes Questionnaire - Short Form 2006 for Brazil. Rev. Latino-Am. Enfermagem. 2012;20(3):575-582.

5. CESTARI VRF, et al. Insuficiência cardíaca: interface com a vulnerabilidade em saúde. Curitiba: Editora CRV, 2019.

6. CESTARI VRF, et al. The essence of care in health vulnerability: a Heideggerian construction. Rev. Bras. Enferm. 2017;70(5):1112-1116.

7. CLARK B, PRETO N. Exploring the concept of vulnerability in health care. CMAJ. 2018; 190(11):E308-E309.

8. ENANG I, et al. Defining and assessing vulnerability within law enforcement and public health organisations: a scoping review. Health and Just. 2019;7(2):1-13.

9. FERNANDES ADF, et al. A 10-year trend analysis of heart failure in the less developed Brazil. Arq Bras Cardiol. 2020; 114(2):222-31.

10. FLORÉNCIO RS. Vulnerabilidade em saúde: uma clarificação conceitual. [tese]. Fortaleza: Programa de Pósgraduação em Saúde Coletiva: Universidade Estadual do Ceará; 2018.

11. FLORÊNCIO RS, et al. Excessive weight and sociodemographic vulnerability markers in young adult students. Acta Paul Enferm. 2016; 29(4):413-20.

12. JASPER MA. Expert: a discussion of the implications of the concept as used in nursing. J Adv Nurs. 1994; 20(4):76976.

13. LI X. Effects of high-quality nursing care on psychological outcomes in patients with chronic heart failure. Medicine. 2019; 98(41):e17351.

14. MAFFACCIOLLI R, OLIVEIRA DLLC. Challenges and perspectives of nursing care to vulnerable populations. Rev Gaúcha Enferm. 2018; 39:220170189.

15. MENDES KDS, et al. Use of the bibliographic reference manager in the selection of primary studies in integrative reviews. Texto contexto enferm. 2019;28:e20170204.

16. MERMET-BOUVIER P, WHALEN MD. Vulnerability and clinical research: mapping the challenges for stakeholders. Ther Innov Regul Sci. 2020; 54(5):1037-46.

17. PANDOVEZE MC, et al. The concept of vulnerability applied to healthcare-associated infections. Rev Bras Enferm. 2019; 72(1):299-303.

18. PASQUALI L. Psicometria: teoria dos testes na psicologia e na educação. 5a ed. Petrópolis: Vozes, 2013.

19. PASQUALI L. Princípios de elaboração de escalas psicológicas. Rev Psiq Clínica. 1998; 25(5):206-13.

20. POLIT DF, BECK CT. Fundamentos de pesquisa em enfermagem: avaliação de evidências para a prática da enfermagem. 9a ed. Porto Alegre: Artmed, 2019.

21. SEFEROVIC PM, et al. The heart failure association atlas: rationale, objectives, and methods. Eur J Heart Failure. $2020 ; 22(4): 638-45$.

22. SILVA DI, et al. Validação de marcadores de vulnerabilidade de lactentes para disfunções em seu desenvolvimento socioemocional. Rev Latino-Am Enfermagem. 2018; 26:e6087.

23. TIMONET-ANDREU E, et al. Health-related quality of life and use of hospital services by patients with heart failure and their family caregivers: a multicenter case-control study. J Nurs Scholarsh. 2020; 52(2):217-28.

24. YILMAZ MB, MEBAZAA A. Definition and characteristics of the vulnerable phase in heart failure. Medicographia; 2015;37(2):144-7.

25. YU DSF, et al. The effects and cost-effectiveness of an empowerment-based self-care programme in patients with chronic heart failure: a study protocol. J Adv Nurs. 2019; 75(12):3740-8. 